\section{Elective Abdominal Aortic Aneurysm Repair Complicated with Chylous Ascites}

\section{Sir,}

Postoperative chylous ascites is a rare complication after abdominal aortic aneurysm (AAA) repair, mostly encountered after surgeries for inflammatory or ruptured aortic aneurysms. ${ }^{1}$ In an open AAA repair, lymphatic vessels can be disrupted. Very few cases have been reported in literature of this condition. ${ }^{2}$

We present a patient who underwent AAA repair and developed chylous ascites. He was managed conservatively.

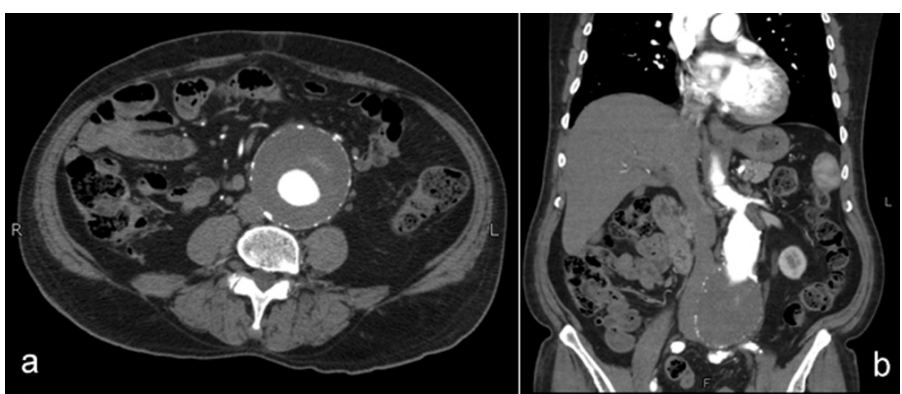

Figure 1 (a,b): Infra-renal abdominal aortic aneurysm.

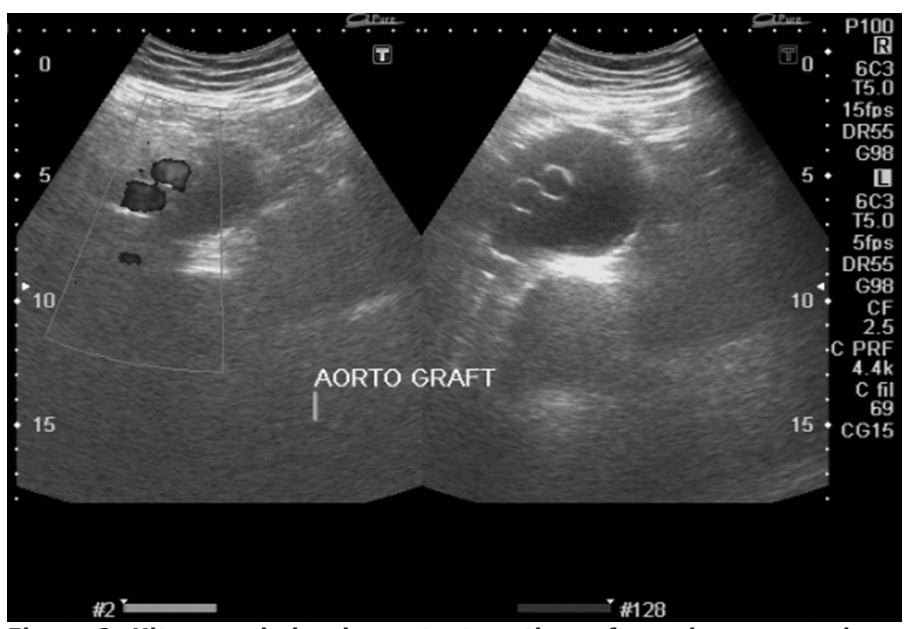
Figure 2: Ultrasound showing patent aortic grafts and aneurysmal sac around them

A 78-year male, cigarette smoker and hypertensive, presented to the clinic with aorto-iliac aneurysm. He was not taking any medications and his family history was insignificant. A computed tomography (CT) angiography was performed, which showed aneurysm measuring $72 \mathrm{~mm}$ in maximum diameter (Figure 1). The aneurysm was also involving left common iliac artery. He underwent elective aortic aneurysm repair. Midline laparotomy was performed and transperitoneal approach was used. The aneurysm was repaired with $10 \times 20 \mathrm{~mm}$ knitted polyester bifurcated graft. One limb was anastomosed to the femoral artery, while otherto the right common iliac artery. There were no intraoperative surprises. Immediate postoperative recovery was smooth. Patient was discharged after uneventful recovery on $10^{\text {th }}$ postoperative day.
Three weeks after the operation, the patient presented to the emergency room with progressively increasing abdominal distension, which was painless and was not associated with fever, constipation, vomiting, breathing difficulty or limb pain. The patient was vitally stable. Abdomen was distended with everted umbilicus. It was soft, non-tender and had a positive fluid thrill. Rest of systemic examinations were normal. Ultrasound of abdomen showed ascites, mostly in the right paracolic gutter and perihepatic region. Under aseptic measures, ascites was aspirated, which showed white, greasy fluid consistent with chyle. It was then confirmed by chemical analysis (Table I). Under local anaesthesia, an $8 \mathrm{Fr}$. pigtail catheter was placed in the right lower abdomen and about 3 litres of milky fluid was drained over the next days. The patient got symptomatically better after drainage (Figure 2). During hospital admission, patient was kept on a lowfat diet. The patient was discharged after one week and followed up in the clinic for two years. He did not have recurrent symptoms.

Table I: Chemicalanalysis of ascitic fluid.

\begin{tabular}{|l|c|}
\hline Test & Result \\
\hline Ascitic fluid glucose & $127 \mathrm{mg} / \mathrm{dl}$ \\
\hline Ascitic fluid protein & $3000 \mathrm{mg} / \mathrm{dl}$ \\
\hline Ascitic TLC & $1900 / \mathrm{cu} \mathrm{mm}$ \\
\hline Ascitic poly & $20 \%$ \\
\hline Ascitic lym & $80 \%$ \\
\hline Ascitic fluid RBC & $(+)$ \\
\hline
\end{tabular}

Chylous ascites accounts for a very small number of overall ascites cases. It is estimated to be 1 case per 20,000 admissions. ${ }^{3}$ The development of chylous ascites after abdominal aortic reconstruction is even rarer.

Lymphatics from lower half of the body join to form lymphatic trunks and that drain into the thoracic duct. The thoracic duct is the largest lymphatic channel in the body. It originates from the cisterna chili, which is a sacculated structure located anterior to the bodies of the first and second lumbar vertebrae. The thoracic duct crosses the aortic hiatus of the diaphragm to enter the right posterior mediastinum. During dissection of the aorta, especially around aortic hiatus, this can get disrupted. If it is significant or postoperative spontaneous closure does not occur, then the patient can present with chylous ascites as was in this case. There are significant anatomical variations in the course and termination of the thoracic duct.

The mainstay of treatment in patients with mild to moderate ascites is a medium-chain triglyceride diet to decrease chyle formation. For severe cases, however, complete bowel rest and total parenteral nutrition must be instituted. Repeated paracentesis results in complete resolution of symptoms in most patients. This can be with or without the use of somatostatin, octreotide and diuretics. The role of surgery comes for refractory ascites not responding to the conservative measures. A preoperative or intraoperative lymphangiogram helps to localise the leak. ${ }^{4}$ Surgical exploration after ingestion of fatty meal and closure of the site of lymphatic injury can be performed. Large mesenteric or paraaortic lymphatic channels can be ligated or oversewn. 
Traditionally, lipiodal lymphangiography is used to identify lymphatic disruption. It can also be used to treat this condition. It is thought that leakage of lipiodol occurs at the site of leakage and initiate local inflammatory process. It closes the lymphatic leakage. It has been shown to be effective in a couple of cases. ${ }^{5}$

\section{CONFLICT OF INTEREST:}

Authors declared no conflict of interest.

\section{AUTHOR'S CONTRIBUTION:}

ZUR: Study concept, data collection, analysis, writing initial draft, critical review, approval of final draft and responsibility of all aspects of work.

\section{REFERENCES}

1. Galanopoulos G, Konstantopoulos T, Theodorou S, Tsoutsas I, Xanthopoulos D, Kaperonis E, et al. Chylous ascites following open abdominal aortic aneurysm repair: An unusual complication. Methodist Debakey Cardiovasc J 2016; 12: 119-21.

2. Olthof E, Blankensteijn JD, Akkersdijk GJ. Chyloperitoneum following abdominal aortic surgery. Vascular 2008; 16: 258-62.

3. Lizaola B, Bonder A, Trivedi HD, Tapper EB, Cardenas A. The diagnostic approach and current management of chylous ascites: A review article. Aliment Pharmacol Ther 2017; 46: 816-24.

4. Al-Busafi SA, Ghali $P$, Deschênes $M$, Wong P. Chylous ascites: Evaluation and management. ISRN Hepatol 2014; 2014: 240-50.

5. Nakamura K, Nakayama K, Minamoto T, Ishibashi T, Ohnishi $\mathrm{K}$, Yamashita $\mathrm{H}$, et al. Ultrasound-guided intranodal lymphangiography with lipiodol for treatment of chylous ascites following surgery for ovarian cancer: A case report. Mol Clin Oncol 2018; 9:178-80.

Zia Ur Rehman

Department of Surgery, The Aga Khan University Hospital, Karachi, Pakistan

Correspondence to: Dr. Zia Ur Rehman, Department of Surgery, The Aga Khan University Hospital, Stadium Road, Karachi, Pakistan

E-mail: ziaur.rehman@aku.edu

Received: February 21, 2020; Revised: May 24, 2020; Accepted: May 24, 2020

DOI: https://doi.org/10.29271/jcpsp.2020.05.557 